\title{
Optical nanofiber coupler sensors operating in the cut-off wavelength region
}

\author{
Wanvisa Talataisong, Rand Ismaeel, Timothy Lee, Martynas Beresna and Gilberto Brambilla
}

\begin{abstract}
An ultrasensitive optical nanofiber coupler (NFC) for thermal and refractive index sensing with nanoscale dimension is proposed and experimentally demonstrated. The NFC operating near the cut-off region of the higher order modes is fabricated by fusing and pulling two standard telecom fibers using the modified flame brushing technique. Due to the submicron diameter, the NFC exhibits an anomalous behavior in close proximity to the odd supermode cut-off wavelength, where the high extinction ratio spectral oscillations exhibit very slow oscillations. The sensitivity of this region is exploited for thermal sensing over a broad range of temperatures $\left(84-661^{\circ} \mathrm{C}\right)$. An average temperature sensitivity of $55 \mathrm{pm} /{ }^{\circ} \mathrm{C}$ and a maximum sensitivity of $60 \mathrm{pm} /{ }^{\circ} \mathrm{C}$ at the highest temperature were demonstrated. Owing to its small size, the thermal sensor exhibits a fast response time of $\sim 7.2 \mathrm{~ms}$. The sensor capability for detecting refractive index changes is also examined with different ethanol concentrations. A record sensitivity of $4.80 \times 10^{5} \mathrm{~nm} / \mathrm{RIU}$ was achieved, which is the highest reported value in all optical fiber refractometers.
\end{abstract}

Index Terms - nanofiber coupler; temperature sensor; refractive index sensor; broad temperature; large dynamic range

\section{INTRODUCTION}

$\mathrm{T}$ he fused optical fiber coupler is a relatively simple passive component, which due to its high performance and low cost, is widely exploited in telecommunications for optical power splitters, various types of interferometers, and coarse wavelength division multiplexing. In the last decade, the optical fiber coupler has been also applied to sensing applications such as pressure [1], micro fluidic flow meter [2], force [3], magnetic field [4], ultrasound [5, 6] and temperature $[7,8]$. This optical sensor benefits from immunity to electromagnetic interference, resistance to corrosion, high sensitivity, and possibility to work in contact with flammables and explosives.

Thermal sensing is one of the areas where fiber couplers could be particularly useful. Optical fiber thermometry has

Manuscript received November 10, 2017; revised December 15, 2017; accepted February 1, 2017. Date of publication Month day, 2018; date of current version Month day, 2018. W. Talataisong received her student scholarship by the Development and Promotion of Science and Technology Talents Project (Royal Government of Thailand scholarship).

Wanvisa Talataisong, Rand Ismaeel, Timothy Lee, Martynas Beresna, and Gilberto Brambilla are with the Optoelectronics Research Centre (ORC), Southampton, Hampshire, SO17 1BJ UK (e-mail: w.talataisong@soton.ac.uk; rmni1g10@orc.soton.ac.uk; t1305@ soton.ac.uk; m.beresna@ soton.ac.uk; g.brambilla@soton.ac.uk).

Digital Object Identifier 10.5258/SOTON/D0296 been investigated for many applications including in the oil and gas industries, electric circuits health monitoring and structural health monitoring. Photonic crystal fibers (PCF) [9, 10], polarization maintaining fibers [11], Fabry-Perot cavities [12], and in-line modal interferometers [13] have been proposed for thermal sensing. However, practical applications are severely limited by complex fabrication processes and high cost. Thus, fiber Bragg gratings (FBG) and long period gratings (LPG) are the most commonly and widely used optical fiber point temperature sensors [14, 15], despite exhibiting a relatively slow response time owing to the large size. Recently, optical microfibers have been investigated for thermal sensing because of their compactness, quick manufacturing, high sensitivity, and fast response time [7, 8 , $16]$.

Another area full of potential for optical fiber couplers is refractometry, which is widely used in metrology, environmental monitoring, chemical and bio-medical sensing. Similar to temperature detectors, several types of optical fiber refractometers have been investigated, such as FBGs, LPGs, PCFs and in-line fiber interferometers [17, 18]. In recent years, refractometers based on microfibers and microresonators have been investigated with outstanding sensitivity, including the microsphere [19], microfiber loop [20] and microfiber coil resonators [21]. However, they exhibit a low sensitivity per refractive index unit (RIU), typically $40-800 \mathrm{~nm} / \mathrm{RIU}$. The optical microfiber coupler (MFC) with a waist diameter of few microns, has shown an immense potential for sensing applications owing to its high evanescent field and large transmissivity changes [22]. Reducing the coupler waist diameter to hundreds of nanometers exponentially increases the evanescent field, thus the coupler interaction with the surrounding environment.

In this paper, we demonstrate a compact high sensitivity thermometer and refractometer based on a nanofiber coupler (NFC). The NFC operates in the odd supermodes cut-off regime, allowing it to maintain high sensitivity over a large range of temperatures and measure minute changes in ambient refractive index.

\section{PRINCIPLE AND FABRICATION}

A typical NFC is composed of two input ports $\left(\mathrm{P}_{1}\right.$ and $\left.\mathrm{P}_{2}\right)$ and two output ports $\left(\mathrm{P}_{3}\right.$ and $\left.\mathrm{P}_{4}\right)$ as shown in Fig. 1, a coupling region of uniform waist formed by two fused parallel nanofibers and two transition regions which connect the waist to the input and output ports. When light is launched into one 
of the input ports, both even and odd supermodes are simultaneously excited. In the coupling region, the wavelength dependent beating of supermodes leads to a continuous change in the power distribution along the coupler cross section, which results in a different power splitting at the output ports for different optical paths.

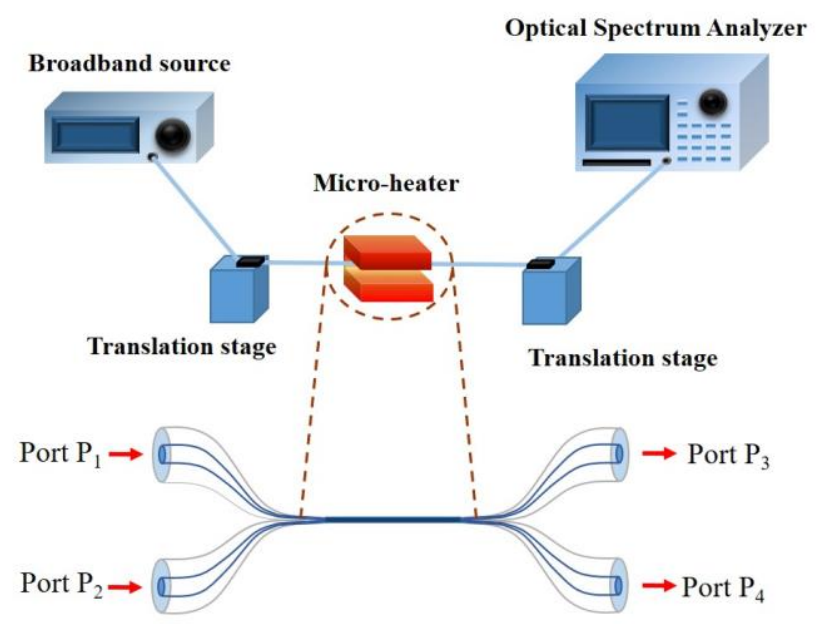

Fig. 1. Schematic of a bi-conical fiber coupler and the experimental setup used to fabricate and test the nanofiber coupler (NFC).

Although the coupler supports multiple supermodes, only the first two are excited, resulting in the singlemode operation at its fiber pigtails. When the diameter (d) of a single nanofiber at coupler waist is smaller than 1 micron, only the first and second supermodes exist in the coupler and at $\mathrm{d} \sim$ $560 \mathrm{~nm}$ only one supermode is supported in the third telecom window (Fig. 2).

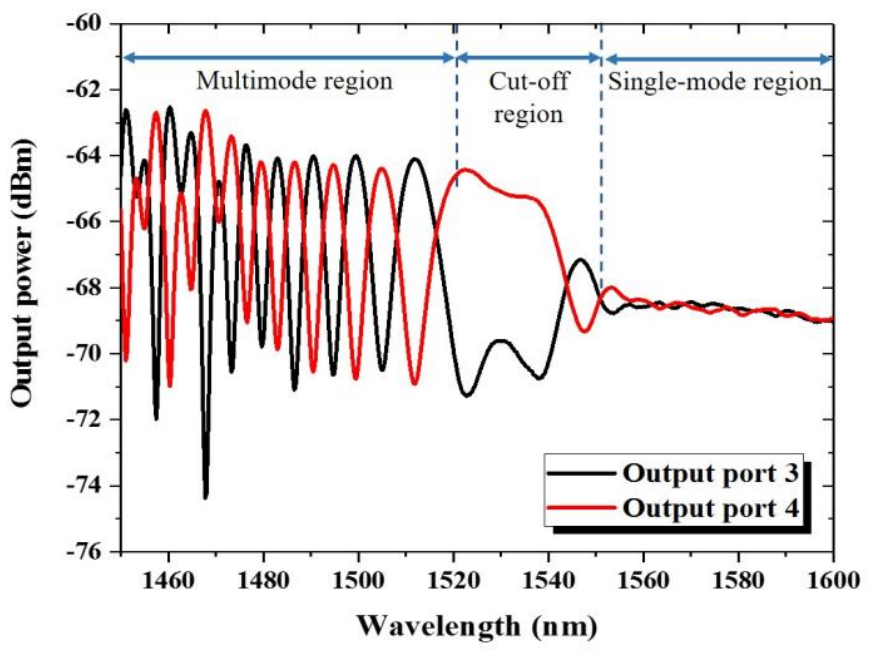

Fig. 2. Output power spectrum of the optical nanofiber coupler with a waist diameter of $560 \mathrm{~nm}$. Light is launched from port 1.

In the multimode region at $\lambda<1520 \mathrm{~nm}$, the beating between two supermodes gives oscillations with high extinctio ratios. In the cut-off region $(1520 \mathrm{~nm}<\lambda<1560 \mathrm{~nm})$, the two supermodes stop beating as one of the supermodes is weakly guided due to the small diameter of the coupler and therefore the two supermodes have a very large difference between their propagation constants $\beta_{\text {even }} \neq \beta_{\text {odd. }}$. Furthermore, since the odd supermode has a much larger fraction of its power in the evanescent field, it is excited less efficiently than the even supermode. For this reason, the power is not distributed equally between the even and odd supermodes and so full power exchange during beating is not possible, leading to a very small dip at $1530 \mathrm{~nm}$ in Fig. 2 and a relatively flat transmissivity over nearly $40 \mathrm{~nm}$. At longer wavelengths, only one supermode is supported by the NFC, resulting in a constant output at both ports, as shown in the single-mode region in Fig. 2.

The NFC can be seen as a weakly fused coupler, approximated as two touching cylindrical waveguides. In this case, the coupling coefficient for the $\mathrm{X}$ polarization $\left(C_{x}\right)$ and Y polarization $\left(C_{y}\right)$ are given by [23]:

$$
\begin{aligned}
& C_{x}+C_{y}=\frac{2^{7 / 2}\left(n_{1}^{2}-n_{0}^{2}\right)^{1 / 2} U_{\infty}^{2}}{n_{1} a(\sqrt{\pi}) V^{5 / 2}} \\
& C_{x}-C_{y}=\frac{2^{5 / 2}\left(n_{1}^{2}-n_{0}^{2}\right)^{1 / 2} U_{\infty}^{2}}{n_{1}^{3} a(\sqrt{\pi}) V^{7 / 2}}
\end{aligned}
$$

where $a$ is the nanofiber diameter, $U_{\infty}=2.405$ and $V=[(2 \pi a) / \lambda]\left(n_{1}^{2}-n_{0}^{2}\right)^{1 / 2}$ and $n_{1}$ and $n_{0}$ are the refractive indices of the silica and ambient air, respectively.

If the injected light at input port 1 in the NFC is unpolarized, the normalized power at an output port is described by:

$$
P=\frac{1}{2}\left[1+\cos \left(\left(C_{x}+C_{y}\right) \cdot L\right) \cos \left(\left(C_{x}-C_{y}\right) \cdot L\right)\right]
$$

where $L$ is the coupling region length of the NFC.

The modal optical paths depend both on the coupling region length and supermodes effective indices, which in turn are affected by the refractive index of the surrounding medium and by the temperature. That provides this structure its unique sensitivity for both temperature and refractive index changes.

The NFC was fabricated from two telecom fibers (Corning SMF-28e, United States) using the microheater brushing technique [24]. The two single mode fibers were stripped of their acrylic coating over a length of $\sim 50 \mathrm{~mm}$ and twisted 1.5 turns around each other. The twisted region was fused together using a resistive ceramic microheater (NTT-AT, Japan) with an estimated temperature of $\sim 1450{ }^{\circ} \mathrm{C}$. An optical microscope image and the scanning electron microscope (SEM) image of the NFC are shown in Fig. 3(a, b): the length of the uniform waist region of fabricated NFC was $4 \mathrm{~mm}$ with a coupler waist diameter of $\sim 1120 \mathrm{~nm}$, providing a single nanofiber diameter of $\sim 560 \mathrm{~nm}$. 
(a)

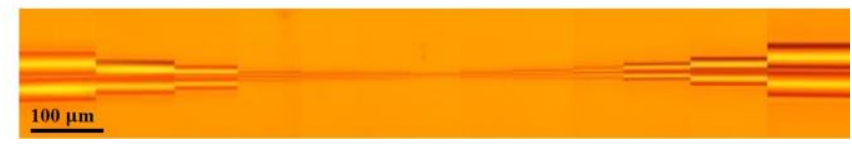

(b)

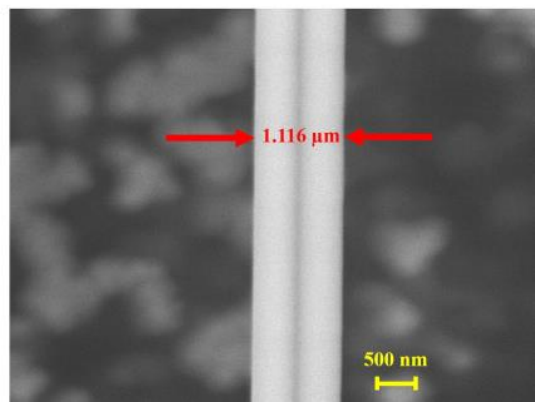

Fig. 3. (a) Microscope image of the NFC with the uniform waist diameter of $560 \mathrm{~nm}$. (b) SEM image at the waist region.

\section{TEMPERATURE MEASUREMENT}

The spectral dependence of the cut-off wavelength region on temperature was investigated positioning the NFC coupling region into the center of the microheater previously used to fabricate the NFC. Light from an incoherent white light source (WLS100, Bentham, U.K.) was launched into port 1 of the NFC and the output of port 3 was connected to an optical spectrum analyzer (AQ6370, Yokogawa, Japan) to monitor the transmission spectrum in real time. The microheater temperature was changed by increasing the current from $0.2 \mathrm{~A}$ to $1.2 \mathrm{~A}$ in steps of $0.1 \mathrm{~A}$, which caused an increase of the microheater temperature from $\mathrm{T}=84{ }^{\circ} \mathrm{C}$ to $661{ }^{\circ} \mathrm{C}$. Temperature calibration of the system was previously performed using a thermocouple positioned in the center of the microheater while the current was gradually increased and decreased. In the NFC experiments, the current was maintained for 15 minutes at each step to ensure that the temperature within the microheater was fully stabilized before being measured.

To evaluate the sensitivity of the nanofiber coupler to temperature, the three transmission wavelength dips which are closest to the cut-off region at the lowest temperature $\left(84^{\circ} \mathrm{C}\right)$ were monitored (Fig. 4). The wavelengths of dip1, dip2, and dip3 are $1505 \mathrm{~nm}, 1495 \mathrm{~nm}$, and $1487 \mathrm{~nm}$, respectively. A red shift of the transmission spectrum can be clearly observed with temperature increase. The refractive index of the silica glass guiding the supermodes in the fiber coupler increases for increasing temperatures due to the thermo-optic effect, therefore, the effective refractive index of the modes supported by the coupler changes. This change causes the shift of the destructive interference responsible for the dips to longer wavelengths. At $\mathrm{T}=478{ }^{\circ} \mathrm{C}$ dip 1 moves into the cut-off region and the weak guidance of the odd supermode results in the disappearance of dip1. Similarly, dip2 disappears at $\mathrm{T}=$ $661^{\circ} \mathrm{C}$.

Results indicate that the wavelength of dip1 can be used to measure temperatures from 84 to $424{ }^{\circ} \mathrm{C}$, while dip2 and dip3 for the temperatures up to $620{ }^{\circ} \mathrm{C}$ and $661{ }^{\circ} \mathrm{C}$, respectively
(Fig. 5). By using a second-degree polynomial function, the slope of dip1 at the highest temperature of $424{ }^{\circ} \mathrm{C}$ was calculated to be equal to $50.80 \mathrm{pm} /{ }^{\circ} \mathrm{C}$ while the slope of dip2 and dip 3 at the highest temperatures were calculated to be equal $60.17 \mathrm{pm} /{ }^{\circ} \mathrm{C}$, and $55.23 \mathrm{pm} /{ }^{\circ} \mathrm{C}$, respectively.

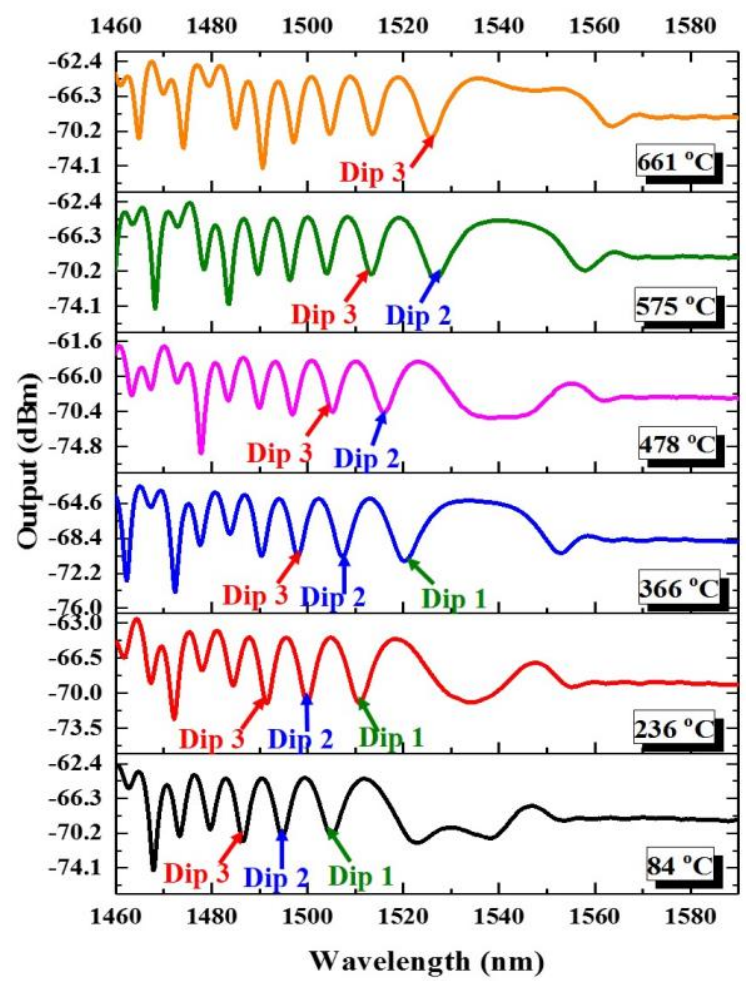

Fig. 4. Optical nanofiber coupler transmission spectrum at different applied temperatures.
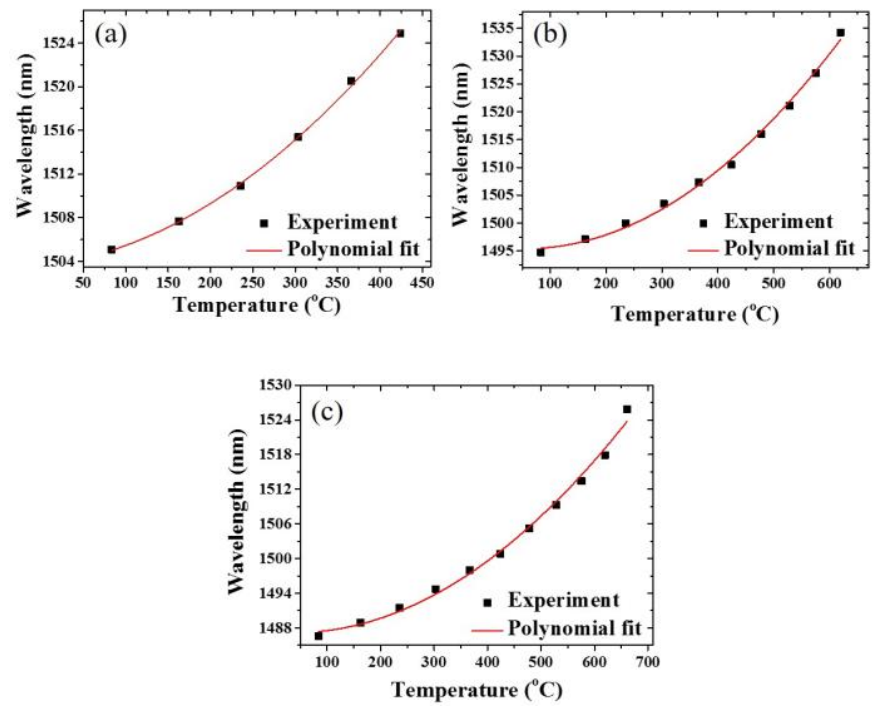

Fig. 5. Relationship between wavelength and applied temperature for different transmission dips (a) dip1 (b) dip 2 (c) dip 3.

To compare the temperature sensitivity of the three wavelength dips, the slope of each graph was measured at a single temperature of $424^{\circ} \mathrm{C}$ (Fig. 6). In the temperature range of $0-424^{\circ} \mathrm{C}$, the sensitivity of dip1, dip2, and dip3, are 50.80 $\mathrm{pm} /{ }^{\circ} \mathrm{C}, 37.21 \mathrm{pm} /{ }^{\circ} \mathrm{C}$, and $33.14 \mathrm{pm} /{ }^{\circ} \mathrm{C}$, respectively. 
The result shows that spectral features at longer wavelengths, especially those in close proximity to the cut-off region, exhibit a higher temperature sensitivity than those at shorter wavelengths and can be used for thermometry. Yet, wavelength dips closer to the cut-off region present a smaller dynamic range.

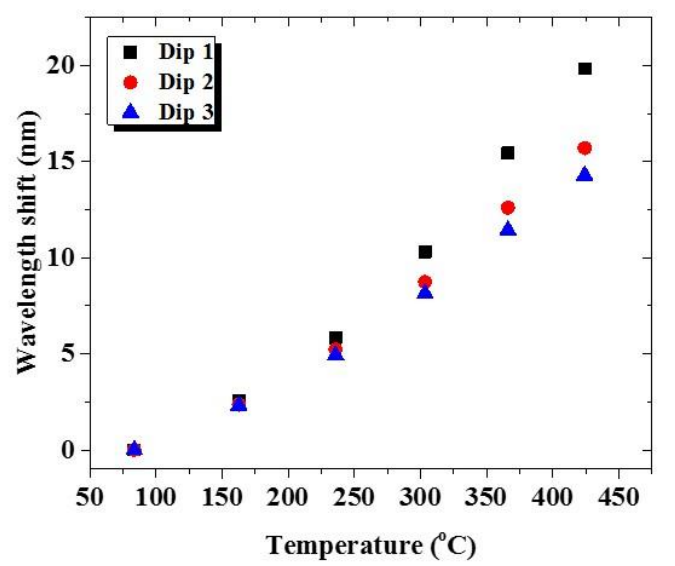

Fig. 6. Relationship between wavelength shift and applied temperature from $84-424{ }^{\circ} \mathrm{C}$ for different transmission dips (a) dip1 (b) dip 2 (c) dip 3.

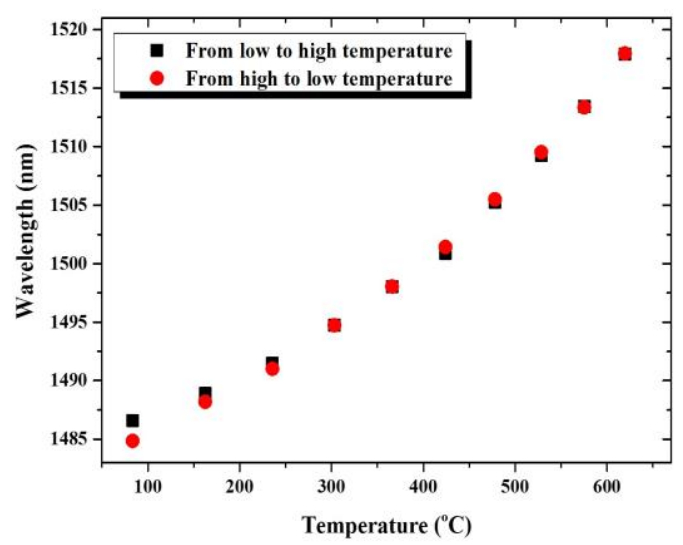

Fig. 7. The wavelength of transmission dip 3 with the variation of microheater temperature. The red dots and the black dots report measurements for decreasing and increasing temperatures, respectively.

The repeatability of the NFC thermometer was measured by recording the output spectra for increasing and decreasing temperatures with an interval of one hour. The wavelength shift of the wavelength of dip 3 with the temperature change is shown in Fig. 7. Results indicate that the NFC thermometer does not exhibit any hysteresis and has a good repeatability.

The response time of the NFC thermometer was also evaluated. The transmission spectrum of the NFC with waist diameter of $\sim 560 \mathrm{~nm}$ for decreasing temperatures is presented in Fig. 8(a) and shows a transmission dip at the wavelength $\lambda$ $=1496.5 \mathrm{~nm}$ at room temperature (blue dash curve) and $\lambda=$ $1499 \mathrm{~nm}$ at the temperature of $661^{\circ} \mathrm{C}$ (red solid curve). When the microheater current is reduced, the temperature decreases and the transmission wavelength dip shifts to shorter wavelengths. Temperature monitoring can be carried out by evaluating the power change over a bandwidth of $1 \mathrm{~nm}$ at a fixed wavelength. An Optical Spectrum Analyzer (OSA) was used as a replacement for a passband filter and a power meter at $\lambda=1500 \mathrm{~nm}$. Figure 8(a) shows that when the temperature was decreased, the recorded intensity at $\lambda=1500 \mathrm{~nm}$ increased.

The thermometer response time was measured by rapidly moving the microheater out from the NFC; the driver current was set at $1.2 \mathrm{~A}$ corresponding to $T \sim 661{ }^{\circ} \mathrm{C}$ (Fig. 8(b)). The response time was obtained considering the time interval at which the intensity increase reached $1 / \mathrm{e}$ of its total change. For the NFC with the waist diameter of $\sim 560 \mathrm{~nm}$ the experimental result of the response time was $7.2 \mathrm{~ms}$.
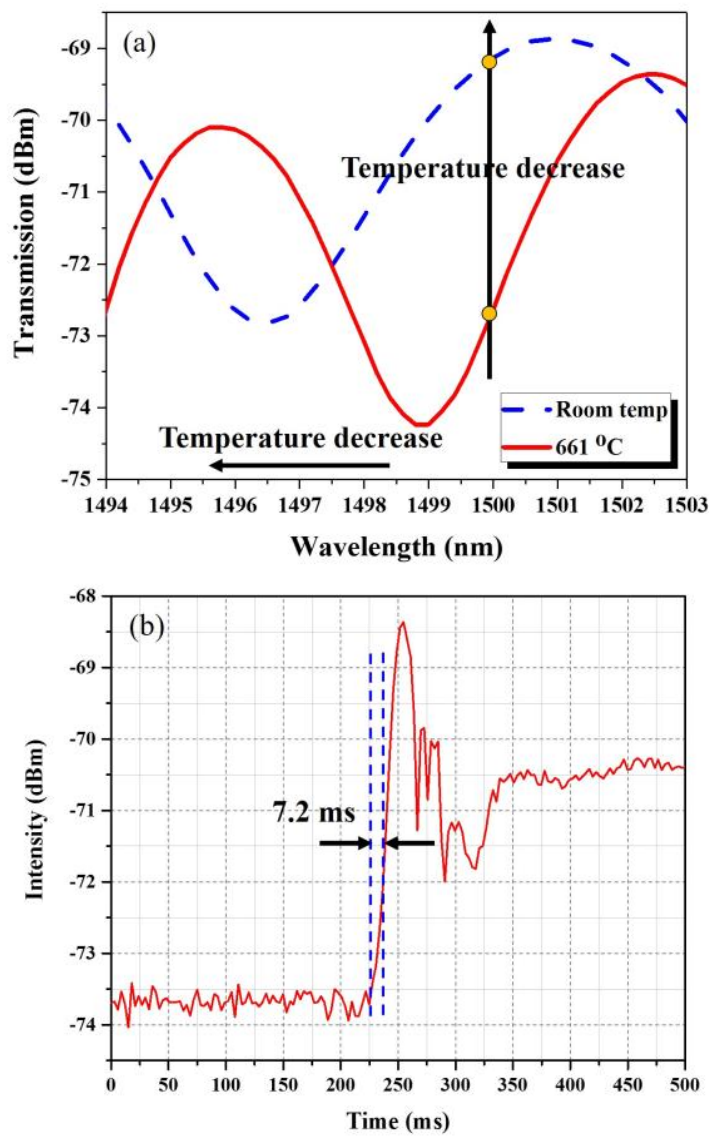

Fig. 8. (a) NFC transmission spectrum at two different temperatures. (b) Temporal dependence of the NFC transmitted intensity recorded for a rapid temperature change.

\section{REFRACTIVE INDEX MEASUREMENTS}

In the refractive index measurements, the waist region of NFC was immersed in $100 \mathrm{ml}$ of distilled water. As before, light from an incoherent white light source was launched into port 1 of the NFC and the output of port 3 was connected to an optical spectrum analyzer to monitor the transmission spectrum in real time. The refractive index (RI) of the solution was increased by adding ethanol into the distilled water by 20 $\mu l$ at each step. In the experiment, the broad peak in the transmission spectrum was monitored with the changing of ambient RI to achieve a large dynamic range.

The result shows that the transmission spectrum of the NFC shifted to shorter wavelengths with increasing ambient RI 
(Fig. 9). To measure RI sensitivity of the sensing device, the RI of an ethanol solution was calculated from the calibration curve of ethanol and water mixture as presented in Fig. 10(a) [25]. Figure 10(b) shows the measured spectral response with the ambient RI values which were calculated from the equation presented in Fig. 10(a). By fitting the wavelength dip and the ambient RI with linear fit, a slope equal to $-4.80 \times 10^{5}$ $\mathrm{nm} / \mathrm{RIU}$ was calculated, providing an overall refractive index sensitivity of $4.80 \times 10^{5} \mathrm{~nm} / \mathrm{RIU}$.

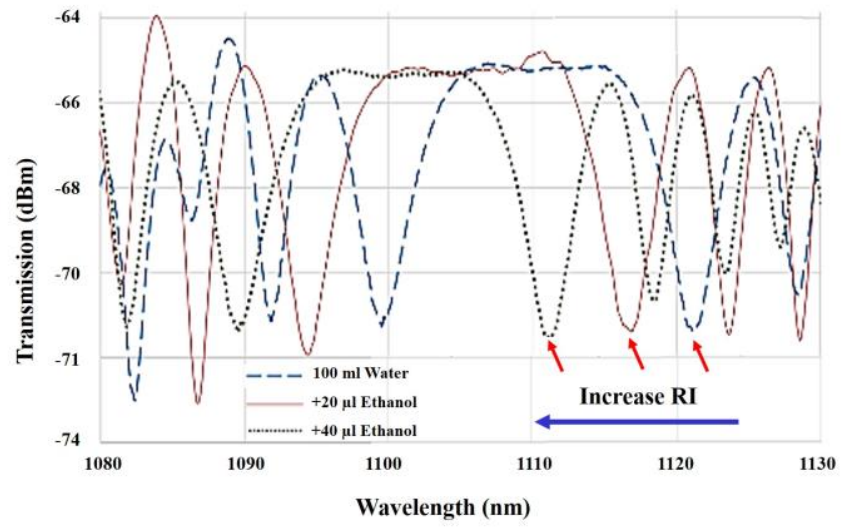

Fig. 9. Change in transmission in the NFC cut-off region at different ambient refractive indexes. Arrows point at the peak used for the sensitivity measurement of fig. 10.
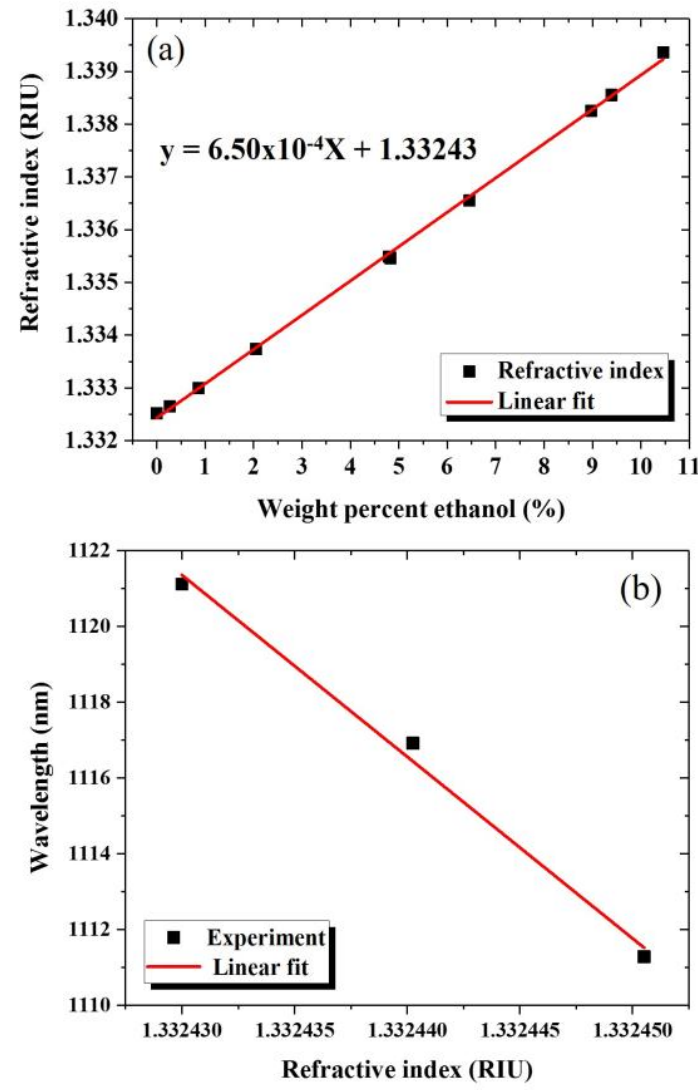

Fig. 10. (a) Calibration curve of the refractive index of the ethanol solution with weight percent of ethanol [25]. (b) Relationship between wavelength dip and the RI value of the surrounding medium.

\section{CONCLUSION}

In conclusion, a thermometer and refractometer based on the cut-off wavelength region of a nanofiber coupler have been investigated. These temperature and refractive index sensors have numerous advantages such as compact size, high sensitivity, and fast response. By monitoring the wavelength of the transmission dips with the temperature increase, a thermometer based on the NFC with the large dynamic range and a high temperature sensitivity of $55.2 \mathrm{pm} /{ }^{\circ} \mathrm{C}$ has been achieved. It efficiently detected high temperatures changes, up to $661{ }^{\circ} \mathrm{C}$ with a response time of $7.2 \mathrm{~ms}$. The temperature sensitivity achieved by using the NFC is approximately 5 times larger than that of sensors based on microfiber couplers. This is a significant improvement compared with earlier demonstrations using larger micron-size fiber coupler thermometers [7]. The NFC also revealed high sensitivity with the ambient refractive index owing to the large evanescent field. A record refractive index sensitivity of $4.80 \times 10^{5}$ $\mathrm{nm} / \mathrm{RIU}$ was obtained. This is the highest reported refractive index sensitivity for all optical fiber based refractometers.

\section{REFERENCES}

[1] L. P. Gafsi R, Malki A., "Stress optical fiber sensor using light coupling between two laterally fused multimode optical fibers.," Apply Optics, vol. 37, no. 16, pp. 9, 1998.

[2] S.-c. Yan, Z.-y. Liu, C. Li et al., ""Hot-wire" microfluidic flowmeter based on a microfiber coupler," Optics Letters, vol. 41, no. 24, pp. 56805683, 2016/12/15, 2016.

[3] Y. Chen, S.-c. Yan, X. Zheng et al., "A miniature reflective micro-force sensor based on a microfiber coupler," Optics Express, vol. 22, no. 3, pp. 2443-2450, 2014/02/10, 2014.

[4] S.-c. Yan, Y. Chen, C. Li et al., "Differential twin receiving fiber-optic magnetic field and electric current sensor utilizing a microfiber coupler," Optics Express, vol. 23, no. 7, pp. 9407-9414, 2015/04/06, 2015.

[5] C. Rongsheng, B. Tim, B. Rod et al., "Linear location of acoustic emission using a pair of novel fibre optic sensors," Journal of Physics: Conference Series, vol. 15, no. 1, pp. 232, 2005.

[6] L. Wang, Y. Liu, W. Fu et al., "Source location using an optimized microfiber coupler sensor based on modal acoustic emission method," Structural Control and Health Monitoring, vol. 24, no. 11, pp. e2011-n/a, 2017.

[7] M. Ding, P. Wang, and G. Brambilla, "A microfiber coupler tip thermometer," Optics Express, vol. 20, no. 5, pp. 5402-5408, 2012/02/27, 2012.

[8] H. Guo, F. Pang, X. Zeng et al., "Temperature sensor using an optical fiber coupler with a thin film," Applied Optics, vol. 47, no. 19, pp. 35303534, 2008/07/01, 2008.

[9] Y. W. C. R. Liao, D. N. Wang, M. W. Yang, "Fiber In-Line MachZehnder Interferometer Embedded in FBG for Simultaneous Refractive Index and Temperature Measurement," IEEE Photonics Technology Letters vol. 22, no. 22, pp. 3, 2010.

[10]Y. S. Jinesh Mathew, Gerald Farrell, "Fiber Optic Hybrid Device for Simultaneous Measurement of Humidity and Temperature," IEEE Sensors Journal, vol. 13, no. 5, pp. 5, 2013.

[11]H.-J. Kim, and Y.-G. Han, "Polarization-Dependent In-Line MachZehnder Interferometer for Discrimination of Temperature and Ambient Index Sensitivities," Journal of Lightwave Technology, vol. 30, no. 8, pp. 1037-1041, 2012/04/15, 2012.

[12]T. Han, Y.-g. Liu, Z. Wang et al., "Simultaneous temperature and force measurement using Fabry-Perot interferometer and bandgap effect of a fluid-filled photonic crystal fiber," Optics Express, vol. 20, no. 12, pp. 13320-13325, 2012/06/04, 2012.

[13]M. Sun, B. Xu, X. Dong et al., "Optical fiber strain and temperature sensor based on an in-line Mach-Zehnder interferometer using thin-core fiber," Optics Communications, vol. 285, no. 18, pp. 3721-3725, 2012/08/15/, 2012 
[14]D. A. Chamorro Enríquez, A. R. da Cruz, and M. T. M. Rocco Giraldi, "Hybrid FBG-LPG sensor for surrounding refractive index and temperature simultaneous discrimination," Optics \& Laser Technology, vol. 44, no. 4, pp. 981-986, 6//, 2012.

[15] Y. Li, M. Yang, D. N. Wang et al., "Fiber Bragg gratings with enhanced thermal stability by residual stress relaxation," Optics Express, vol. 17, no. 22, pp. 19785-19790, 2009/10/26, 2009.

[16]P. Wang, M. Ding, L. Bo et al., "Fiber-tip high-temperature sensor based on multimode interference," Optics Letters, vol. 38, no. 22, pp. 46174620, 2013/11/15, 2013.

[17]K. J. Lee, X. Liu, N. Vuillemin et al., "Refractive index sensor based on a polymer fiber directional coupler for low index sensing," Optics Express, vol. 22, no. 14, pp. 17497-17507, 2014/07/14, 2014.

[18]P. Wang, G. Brambilla, M. Ding et al., "Enhanced Refractometer Based on Periodically Tapered Small Core Singlemode Fiber," IEEE Sensors Journal, vol. 13, no. 1, pp. 180-185, 2013.

[19] N. M. Hanumegowda, Stica, Caleb J, Patel, Bijal C, White, Ian, Fan, Xudong, "Refractometric sensors based on microsphere resonators," Applied Physics Letters, vol. 87, no. 20, pp. 3, 2005.

[20] T. L. Guo X "Supported microfiber loops for optical sensing," Optics Express, vol. 16, no. 19, pp. 6, 2008.

[21]G. Brambilla, and F. Xu, "Demonstration of a Refractometric Sensor Based on Optical Microfiber Coil Resonator," OSA Technical Digest $(C D)$. p. CMJJ5

[22] Y. Jung, G. Brambilla, and D. J. Richardson, "Optical microfiber coupler for broadband single-mode operation," Optics Express, vol. 17, no. 7, pp. 5273-5278, 2009/03/30, 2009.

[23] C. D. H. F.P. Payne, M.S. Yataki, "Polarisation analysis of strongly fused and weakly fused tapered couplers," Electronics Letters vol. 21, no. 13, pp. 3, 1985 .

[24]G. Brambilla, "Optical fibre nanowires and microwires: a review," Journal of Optics, vol. 12, no. 4, pp. 043001, 2010.

[25]A. Troy, Scott Jr," "Refractive index of ethanol-water mixtures and density and refractive index of ethanol-water-ethyl ether mixtures.," $J$ Phys Chem., vol. 50, no. 5, pp. 12, 1946.

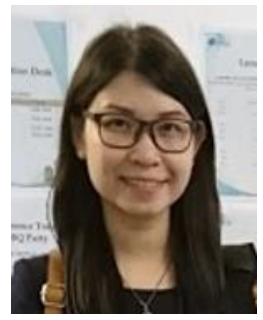

Wanvisa Talataisong received her BSc degree in physics from Khonkaen University, Khonkaen, Thailand, in 2011 and her MSc degree in physics from Mahidol University, Bangkok, Thailand, in 2015. She was an internship student at The Hong Kong polytechnic University, Hong Kong, in 2014. She is currently pursuing her $\mathrm{PhD}$ in optoelectronics at the Optoelectronics Research Centre (ORC), University of Southampton. Her previous research includes the fabrication of mechanical induced ultra-long period fibre grating and optical fibre sensors based on in-line interferometers. Her current research interest includes the fabrication of optical fibre nanosensors and the fabrication of polymeric fibres based on 3D-printed preform.

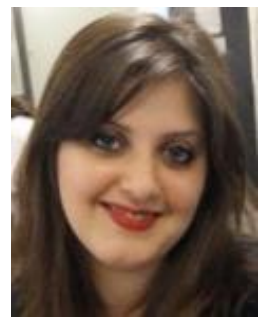

Rand Ismaeel received her BSc in 2008 and MSc in 2009 in laser and optoelectronic engineering from Al Nahrain University (Baghdad). She joined the ORC at the University of Southampton in 2010 and got her PhD in 2015, where she has been working on micro/nanofiber resonators and related sensors. She is currently employed as a Research Fellow within the ORC and her research area divided into major areas of optical microfiber devices and sensors, high power lasers, and nonlinear optics.

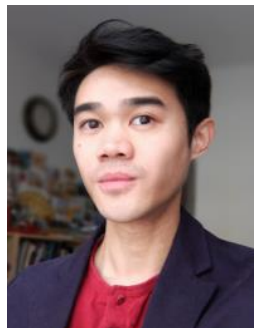

Timothy Lee received his MEng degree in Electronic engineering (2009) and $\mathrm{PhD}$ in Optoelectronics (2013) from the University of Southampton (UK), where he is currently a Research Fellow at the Optoelectronics Research Centre. His doctoral work focused on nonlinear effects in microfibers and microfiber resonators, and post-doctoral research areas include terahertz generation, high power pulsed laser design, and femtosecond writing of waveguide-based devices and lenses.

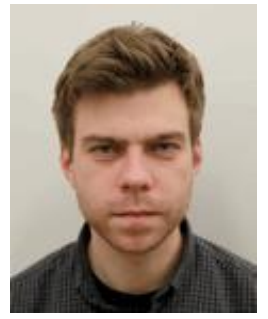

Martynas Beresna obtained a BSc degree in physics in 2008 from Vilnius University, Lithuania. He then joined the Optoelectronics Research Centre at the University of Southampton to undertake his doctoral studies, and he received a PhD degree in optoelectronics in 2012.

Currently, he is holding a senior research fellow position with the Optical Microfibre Devices and Sensors group at the Optoelectronics Research Centre. His research interests include polarization optics, optical fibres and their applications in sensing.

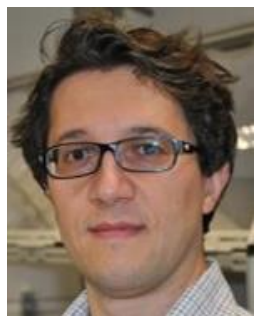

Gilberto Brambilla received his MSc (Hons.) degree in material engineering from Politecnico di Milano, Italy, in 1996 and his $\mathrm{PhD}$ in optoelectronics from the ORC in 2002. He is currently a Professor at the ORC, where he has worked since 2002. In 2007, he received the University Research Fellowship from the Royal Society. Until 2015 he has been director of the EPSRC Centre for Innovative manufacturing in Photonics and he is currently Deputy Director and General manager of The Future Photonics Hub. His research interests include optical fibre devices and sensors, rare earth doped scintillating fibers, special fibers and fiber combiners for high brightness highpower fiber lasers, specialty fibres, UV optical fibre laser, nonlinear optics, femtosecond laser processing, and manufacturing technologies. 